Scientific Review - Engineering and Environmental Sciences (2021), 30 (2), 327-336

Sci. Rev. Eng. Env. Sci. (2021), 30 (2)

Przegląd Naukowy - Inżynieria i Kształtowanie Środowiska (2021), 30 (2), 327-336

Prz. Nauk. Inż. Kszt. Środ. (2021), 30 (2)

http://iks.pn.sggw.pl

DOI 10.22630/PNIKS.2021.30.2.28

Volodymyr SNITYNSKYY ${ }^{1}$, Ivan KERNYTSKYY ${ }^{2}$, Bohdan DIVEYEV ${ }^{3}$, Orest HORBAY $^{3}$, Yevhen FORNALCHYK ${ }^{3}$, Ruslan HUMENUYK ${ }^{1}$, Yaroslav SHOLUDKO ${ }^{1}$

${ }^{1}$ Lviv National Agrarian University - LNAU, Faculty of Mechanical and Power Engineering

${ }^{2}$ Warsaw University of Life Sciences - SGGW, Institute of Civil Engineering

${ }^{3}$ Lviv Polytechnic National University - LPNU, Institute of Engineering Mechanics and Transport

\title{
Optimization of the semi-active vibration absorbers
}

Key words: dynamic vibration absorber, semi-active, adaptive schemes, optimization, robustness, design

\section{Introduction}

Given the diversity of types of dynamic processes that cause increased vibrations and increased operational requirements, the development of methods and means of vibration protection, in particular - passive ones or controlled remains relevant. Among them, a special place is occupied by dynamic vibration absorber (DVA), which are joined to the design in order to reduce its vibration load. A special feature of DVA is that their use can be foreseen at the design and construction stage, and in case of elimination of unsatisfactory characteristics of the structure already in the process of its operation.
DVAs differ in the methods of energy dissipation. The most well-known are DVAs with a viscoelastic element or a combination of an elastic element and a damper. Electromagnetic and electrostrictive processes are also used in all types of DVAs with electromagnets or piezoelectric elements. Such DVAs usually belong to the class of active, because with the help of electrical processes it is easier to control processes.

One of the main directions of the development of the theory of fading oscillations is to evaluate their efficiency and to select optimal parameters with different disturbing loads. To date, the theory of single-mass linear DVA with viscous and independent friction with harmonic oscillations with stable or fixed frequency (which varies in time) is most fully developed.

The semi-active DVA uses control to adjust the device configuration based on the actual structure of the vibration. As 
is typical for structural control, there are many types of devices and management laws, some of them are more realistic than others of varying degrees of complexity. A considerable number of practical implementations of semi-active DVA's can be found in the literature, some of which are described in Seiler, Fischer and $\mathrm{Hu}-$ ber (2002), Casciati, Magonette and Marazzi (2006), Setareh, Ritchey, Murray, Koo and Ahmadian (2007), Nagarajaiah (2009), Shena and Ahmadian (2013), Weber and Distl (2013).

A broad description of the control algorithms that can be used to control these devices can be found in Setareh et al. (2007), which in turn are divided into two groups. Some of them are based on strategies that constantly change the parameters of the DVA in dynamic mode, and the other - on the ON/OFF control strategy. The latter option, though not as impressive as the first one, tends to result in algorithms that are simpler, more realistic, and easier to implement.

For realization of semi-active control various mechanisms for controlling the movement of DVA are offered. The mechanism based on the magneto-rheological (MR) control method has recently gained popularity in the structural control zone (Spencer \& Nagarajaiah, 2003). The application of a magnetic field causes changes in the structure of the liquid MR inside the damper, which is used to control the damping force, in these elements. The simplicity of its mechanical functioning is associated with low direct constructive costs and low running costs for their operation. DVA can simultaneously reduce amplitude of oscillations at several frequencies. In Pinkaew and Fujino (2001) based on the optimal control theory, it has been found that a semi-active DVA can extinguish the vibration of the primary in both the transient and in steady-state modes. In Hu and Jin (1997) and Qian and $\mathrm{Hu}$ (2001) a semi-active vibration absorber with piecewise linear elastic components is presented, which may have an adjustable the operating frequency, which follows the frequency variation of the excitation. In Koo and Ahmadian (2004) numerically studied two kinds semi-active skyhook DVA, and then magnetic resonator (MR) damper is tapped and experimentally confirmed the improved control efficiency. The conventional DVA are presented in Diveyev, Horbay, Kernytskyy, Pelekh and Velhan (2017), Kernytskyy et al. (2017), Pelekh et al. (2017).

\section{Semi-active DVA}

Consider the frequency response and vibrograms of uncontrolled and controlled damping of DVA (Fig. 1).

The DVA equations in this case will be:

$$
\begin{aligned}
& m_{1} \frac{d^{2} x_{1}}{d t^{2}}+k_{1} x_{1}+c_{1} \frac{d x_{1}}{d t}- \\
& -k_{2}\left(x_{2}-x_{1}\right)-c_{2}\left(\frac{d x_{2}}{d t}-\frac{d x_{1}}{d t}\right)- \\
& -c_{t 2} \operatorname{sign}\left(\frac{d x_{2}}{d t}-\frac{d x_{1}}{d t}\right)=k_{1} x_{0}+c_{1} \frac{d x_{0}}{d t}
\end{aligned}
$$

$$
\begin{aligned}
& m_{2} \frac{d^{2} x_{2}}{d t^{2}}+k_{2}\left(x_{2}-x_{1}\right)+c_{2}\left(\frac{d x_{2}}{d t}-\frac{d x_{1}}{d t}\right)+ \\
& +c_{t 2} \operatorname{sign}\left(\frac{d x_{2}}{d t}-\frac{d x_{1}}{d t}\right)=0
\end{aligned}
$$




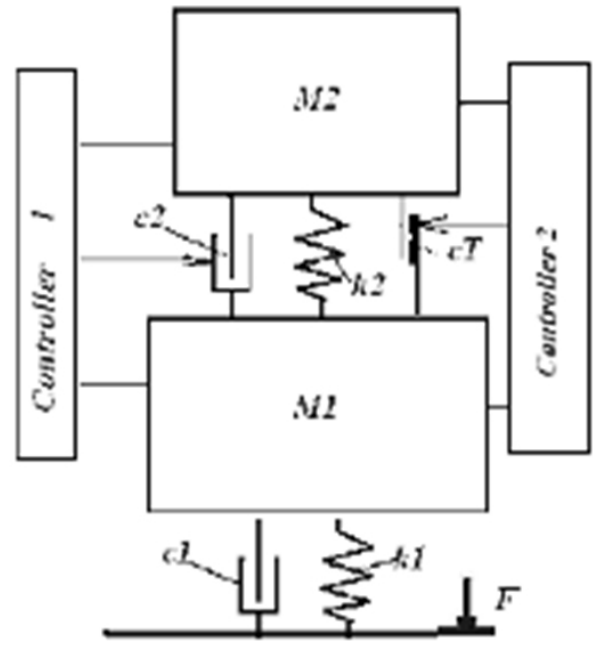

FIGURE 1. Semi-active DVA with the damping and friction control

Here a kinematic perturbation with a certain frequency is considered. The coefficients are now variables in time and change according to some rules with the help of controllers (Fig. 1). Here the rule ON-OFF of semi-active DVA's can be found in the literature, some of which are described in $\mathrm{Hu}$ and Jin (1997), Pinkaew and Fujino (2001), Qian and Hu (2001), Seiler et al. (2002), Spencer and Nagarajaiah (2003), Koo and Ahmadian (2004), Casciati et al. (2006), Setareh et al. (2007), Nagarajaiah (2009), Weber and Distl (2013), for viscous friction is applied:

$$
\begin{aligned}
& \operatorname{sign}\left(x_{1}\left(\frac{d x_{2}}{d t}-\frac{d x_{1}}{d t}\right)\right)>0, c_{2}=c_{2 A} \\
& \operatorname{sign}\left(x_{1}\left(\frac{d x_{2}}{d t}-\frac{d x_{1}}{d t}\right)\right) \leq 0, c_{2}=c_{2 B}
\end{aligned}
$$

The complexity and high dimensionality of some models lead to the use of a heuristic search method.

\section{DVA optimization}

Wide application for optimization found algorithms of random search, namely genetic algorithms (Allen \& Karjalainen, 1999). The problem of solving such problems is the direction in the computer sciences, which is called artificial intelligence (AI). For application of GA mathematical models should adequately reproduce the real dynamic processes in machines and structures with DVA. They must satisfy the following basic requirements:

1. Adequate reproduction of dynamic processes in structures.

2. To include in the set of calculation parameters the determining design and technological parameters.

3. To be adapted to the next transformation into machine codes (there is somewhat absurd idea that designs should be designed not only on the basis of their functional characteristics, but also on the requirements of their maximum simplification, from the conditions of their simplest mathematical modeling).

4. Interact with known software application packages of computer design and computer-based production training.

5. Have an information correlation with world-wide patent knowledge bases (for example, from espacenet.com) and with accessible "open" software on the Internet. There are concepts of local and global object search. The complexity and high dimensionality of some models lead to the use of a heuristic search method. In this matter, random search methods (genetic algorithms - GA) were used to optimize DVA. 
The optimization function is

$$
F_{C I L}=\max \left(\ddot{x}_{1}(f)\right), f_{1}<f<f_{2}
$$

Results of optimization of the frequency response for DVA (frequency response function - FRF) with constant damping and controlled damping are presented in Figure 2a, the corresponding vibration for the frequency inside the optimization interval are presented in Figure 2b. Here $x_{1}$ - vibration level of base, $f_{1}, f_{2}$ - boundaries of observed frequency domain, $P$ - weight function, $f$ - first eigen-frequency. Parameters of optimization are $m_{2}, k_{2}, c_{2}$. Just as a viscous friction, let us consider a variable dry friction (4). $\operatorname{sign}\left(x_{1}\left(\frac{d x_{2}}{d t}-\frac{d x_{1}}{d t}\right)\right)>0, c_{t 2}=c_{T 2 A}$

$$
\operatorname{sign}\left(x_{1}\left(\frac{d x_{2}}{d t}-\frac{d x_{1}}{d t}\right)\right) \leq 0, c_{t 2}=c_{T 2 B}
$$

[The ordinate units are not specified here (as well in Figs. 5, 6, 8, 10) as all problems are linear. The appearance of the graphs will be identical, regardless of the intensity of the load].

In Figure 3 the results of optimization for DVA with controlled viscous damping, friction damping and both simultaneously are shown. In the narrower range, active friction is more effective (Fig. 4).

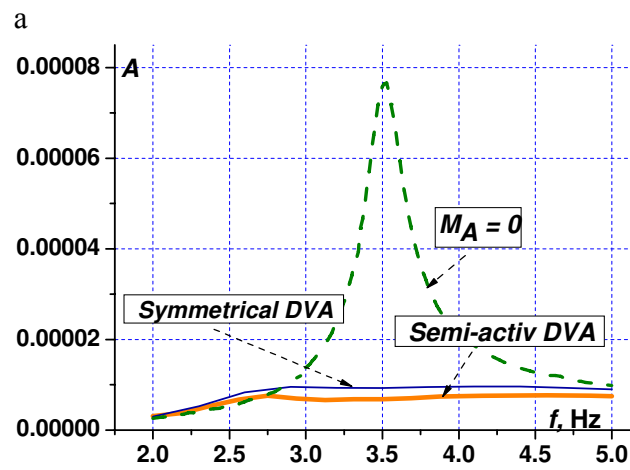

b

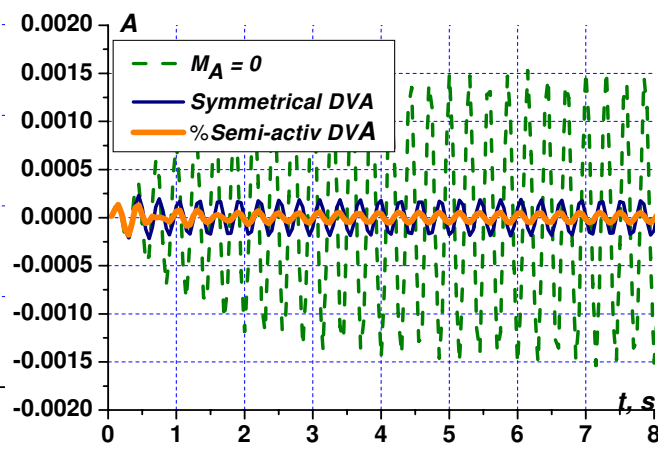

FIGURE 2. Results of optimization of the frequency response for DVA with constant damping and controlled damping (a); the corresponding vibration for the frequency inside the optimization interval (b)

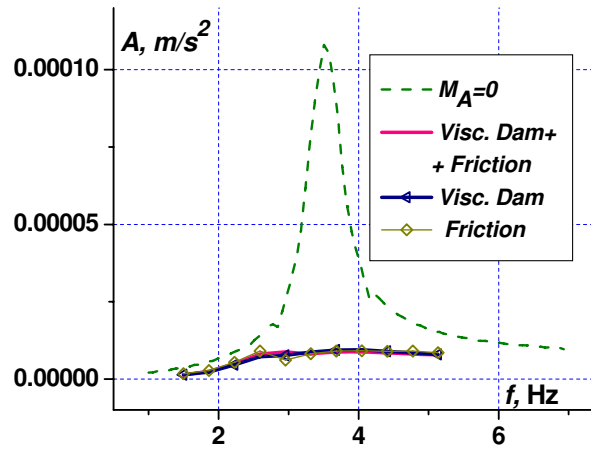

FIGURE 3. Optimized FRF's for controlled viscous, dry friction and both controlled simultaneously

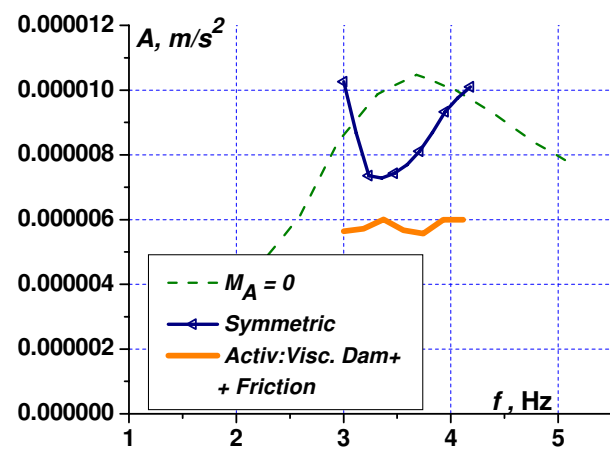

FIGURE 4. Narrower range of optimization 


\section{Pulse optimization}

Consider the optimization of the DVA by parameters, with impulse disturbance

$$
C i L=\max \left(\left|u_{1}(t)\right|\right), t>T_{0}
$$

Here, for the target function, the maximum deviation of the amplitude of the basis is taken after some initial period of time. Figures 5 and 6 show the results of optimization in the control of viscous and dry friction and without control.

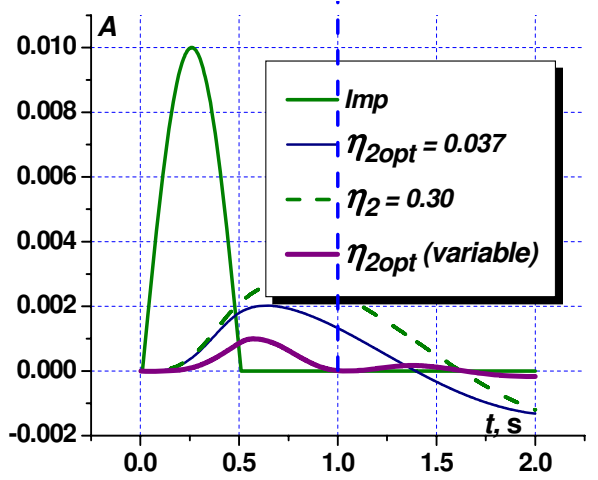

FIGURE 5. Results of optimization in the control of viscous friction and without control

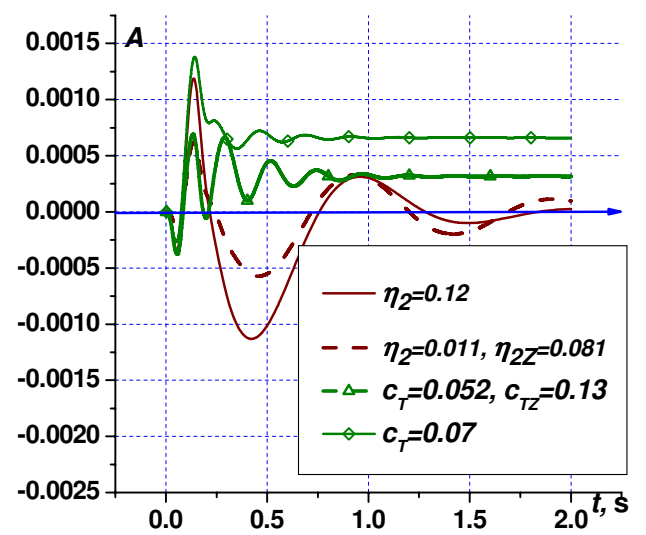

FIGURE 6. Optimization results in the management of viscous and dry friction and without control

\section{DVA robust optimization}

The fundamental definition of robust design is described as a product or process is said to be robust when it is insensitive to the effects of sources of variability, even through the sources themselves have not been eliminated (Fowlkes \& Creveling, 1995). The questions of robustness are discussed in Zang, Friswell and Mottershead (2005) at optimization of DVA. As the main criterion the weighted sum of deviation of the basic design and the gradient module of this deviation is considered. A set of random values of such parameters as mass and stiffness of DVA is used for graphically constructing a shell of optimal DVA with the deviation of parameters.

In our optimization method, genetic methods are used that give a sequence of points of parameters that coincide with the optimal value. On the basis of intermediate results of calculations, it is possible to construct not shells of plane charts, but map parameters, which is more informative. For this purpose, one or two start-ups of the optimization process, preferably with diametrically opposite points of permissible values, prevail. The criterion of robustness is the convexity of these maps in the vicinity of the optimum. Although this is not a sufficient condition, only necessary. For a more complete study, we introduce the following indicator - the radius of deviation $\left(R_{\Delta}\right)$.

$$
R_{\Delta}=\min (R), C i L a=(1+\alpha) C i L
$$

This is the minimum distance from the points with the indicators $(1+\alpha)$ at 
the higher (worst) points from the optimal point. For optimization in a linear case, only two parameters are depicted in Figure 7 for $\alpha=2$. In this case, this condition is also sufficient. In the case of a lot of parametric optimization, the convexity of all maps across all parameters pairs is a necessary, but not sufficient condition. In a multidimensional space, the behavior of the target function can be complex. However, the criterion that finds the nearest point with some deviation of the point to the optimum remains effective. For optimization in a linear case only for two parameters (frequency and damping) are depicted in Figure 7 for $\alpha=2$.

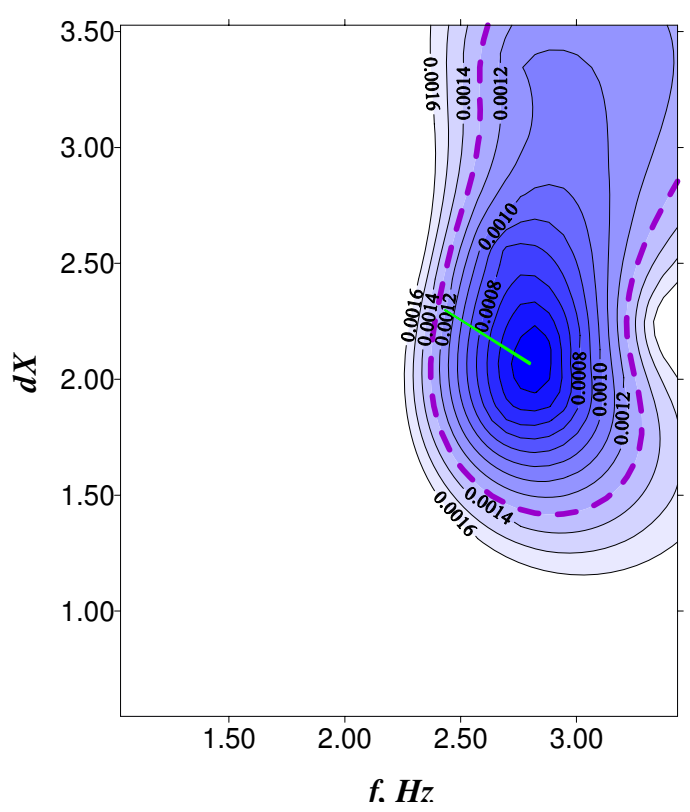

FIGURE 7. Map of optimization in by two parameters, and the indicator of robustness

\section{Different types of control management}

Consider some simple control algorithms. This is, first of all, the rule (2) is known as sky-hook or ON-OFF (Hu \& Jin, 1997; Pinkaew \& Fujino, 2001; Qian \& Hu, 2001; Seiler et al., 2002; Spencer \& Nagarajaiah, 2003; Koo \& Ahmadian, 2004; Casciati et al., 2006; Setareh et al., 2007; Nagarajaiah, 2009; Weber \& Distl, 2013) for viscous friction. The effectiveness of this rule in the wide-frequency range is shown in Figure 7. But there are similar rules of management (Shen \& Ahmadian, 2013; Moutinho, 2015):

$$
\begin{aligned}
& \operatorname{sign}\left(\frac{d x_{1}}{d t}\left(\frac{d x_{2}}{d t}-\frac{d x_{1}}{d t}\right)\right)>0, c_{2}=c_{2 A} \\
& \operatorname{sign}\left(\frac{d x_{1}}{d t}\left(\frac{d x_{2}}{d t}-\frac{d x_{1}}{d t}\right)\right) \leq 0, c_{2}=c_{2 B}
\end{aligned}
$$

or

$$
\begin{aligned}
& \operatorname{sign}\left(\frac{d^{2} x_{1}}{d t^{2}}\left(\frac{d x_{2}}{d t}-\frac{d x_{1}}{d t}\right)\right)>0, c_{2}=c_{2 A} \\
& \operatorname{sign}\left(\frac{d^{2} x_{1}}{d t^{2}}\left(\frac{d x_{2}}{d t}-\frac{d x_{1}}{d t}\right)\right) \leq 0, c_{2}=c_{2 B}
\end{aligned}
$$

We shall also investigate the effectiveness of different control rules in the classical case in the vicinity of the resonance (Fig. 8).

Just like outside the resonance zone, ON-OFF control is effective only. Other management is just a bit better than an uncontrollable process.

Let us consider the process of optimization DVA with the fewer notions 


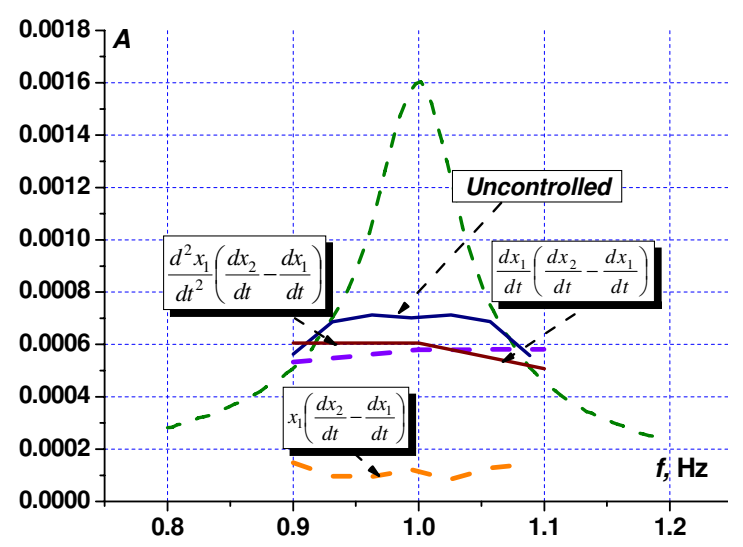

FIGURE 8. The effectiveness of different control rules in the vicinity of the resonance $m_{2} / m_{1}=0.06$ (ON-OFF algorithm). In Figure 9 the process of genetic optimization is shown.

In Figure 10 the results of optimization are shown. In Figure 11 variation of controlled damping and in Figure 12 the map of optimal parameters are presented.

Let us consider less DVA mass $\left(m_{2} / m_{1}=0.02\right)$. In Figure 13 the vibration of base is shown by optimal DVA.

a

$\mathrm{N}=1$

fx.804E+00 dx.560E+00 dx2.254E-01 Av.249E+00 dv.131E+00 CiL.444E-02 $\mathrm{N}=13$

fx.799E+00 dx.682E+00 dx2.243E-01 Av.238E+00 dv.131E+00 CiL.431E-02 $\mathrm{N}=15$

fx.804E+00 dx.682E+00 dx2.169E-01 Av.249E+00 dv.131E+00 CiL.407E-02 $\mathrm{N}=70$

fx.979E+00 dx.532E+00 dx2.191E-02 Av.764E+00 dv.957E-01 CiL.293E-02 $\mathrm{N}=75$

fx.950E+00 dx.716E+00 dx2.379E-02 Av.763E+00 dv.119E+00 CiL.224E-02 $\mathrm{N}=3343$

fx.965E+00 dx.964E+00 dx2.552E-03 Av.344E+00 dv.502E+00 CiL.177E-02 $\mathrm{N}=3544$

fx.966E+00 dx.963E+00 dx2.558E-03 Av.683E+00 dv.502E+00 CiL.173E-02

b

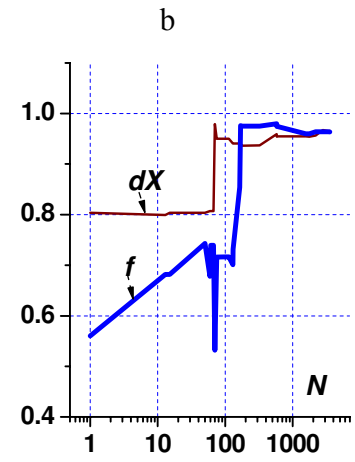

FIGURE 9. The process of genetic optimization is shown: a - step by step date; $\mathrm{b}$ - graphical representation of frequency and damping a

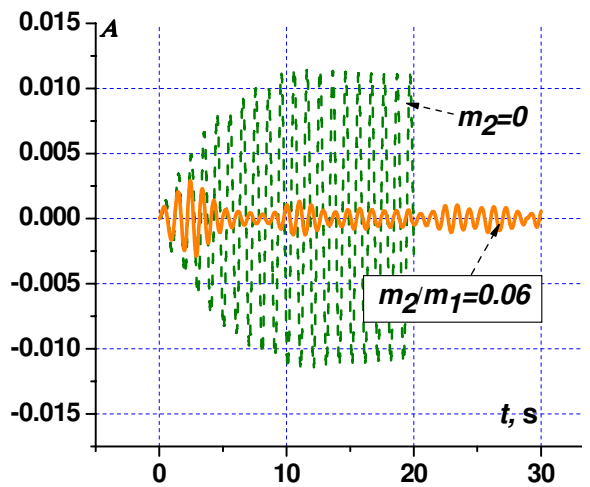

b

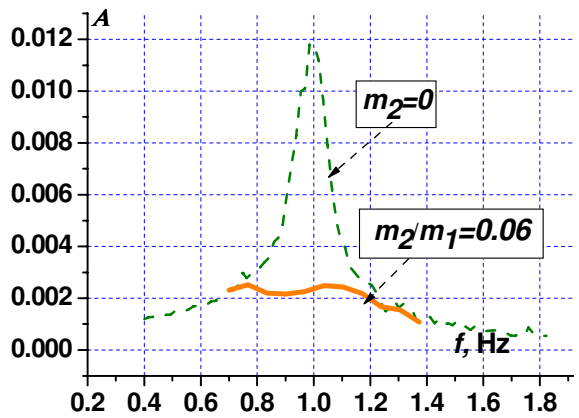

FIGURE 10. Results of optimization: $\mathrm{a}$ - in the time domain; $\mathrm{b}$ - optimal FRF 


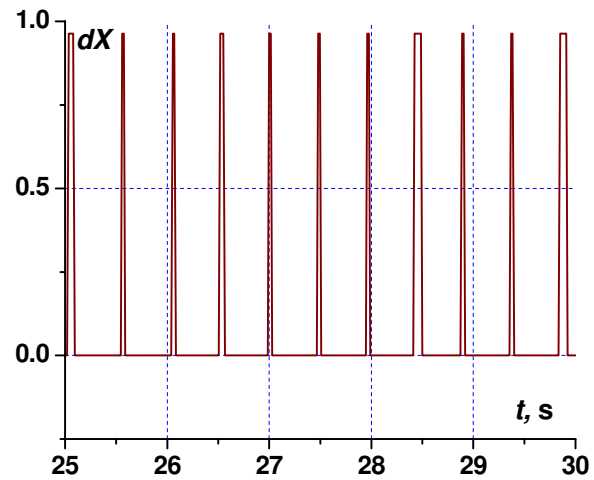

FIGURE 11. Variation of controlled damping
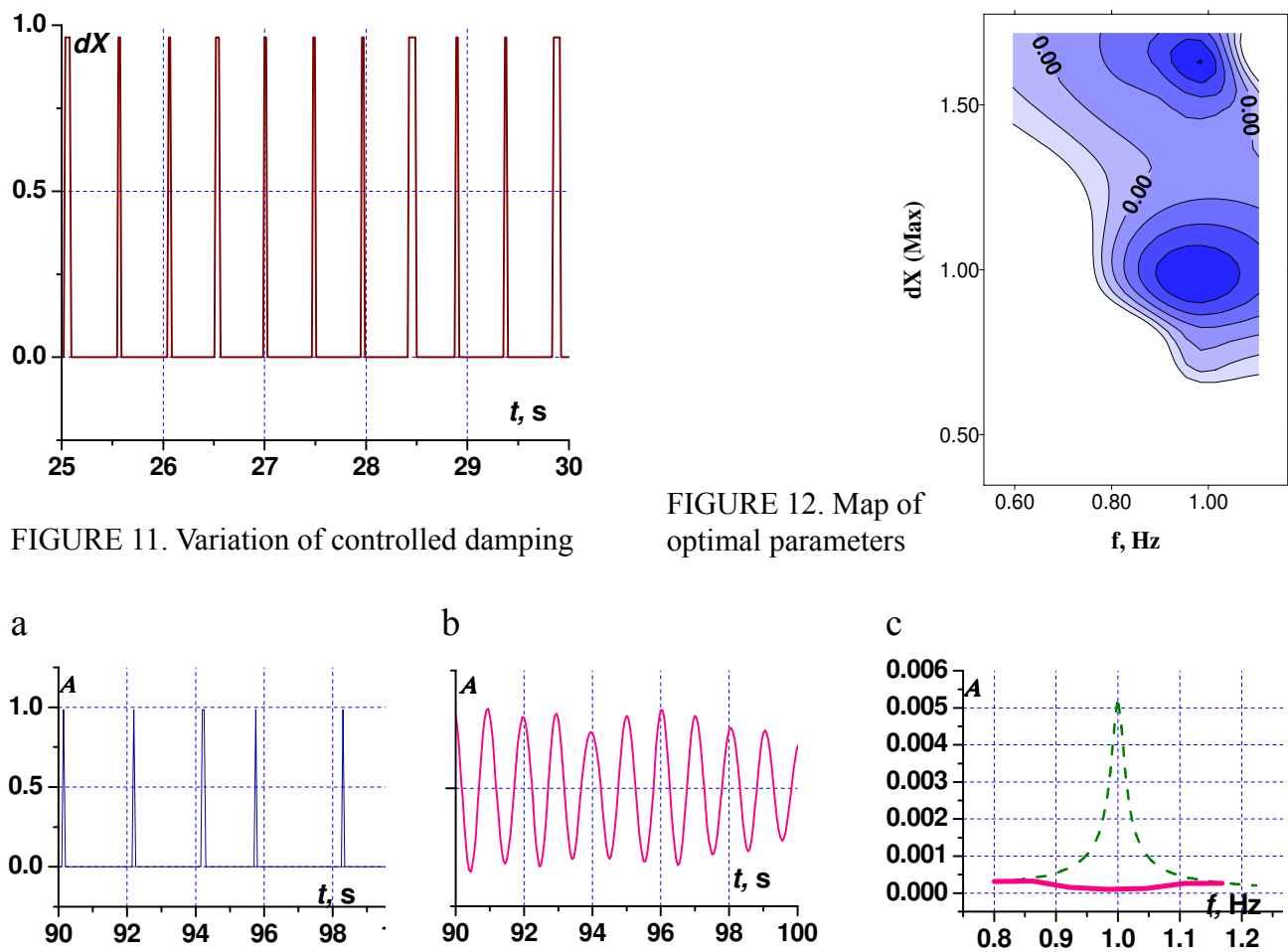

FIGURE 13. Vibration of base: a - changing damping (after $90 \mathrm{~s}$ ); $\mathrm{b}$ - time domain; $\mathrm{c}$ - optimal FRF

\section{Conclusions}

The semi-active DVAs, which are controlled both by damping and frequency, are considered. The new methods of DVA optimization by impact and narrow frequency excitation are considered. On the basis of the simple models, it is possible to optimize different types of DVA in the range of the first resonance of the basis, where, as a rule, the greatest amplitudes of oscillations. Separately, various types of DVA control: skyhook (different types), ON-OFF, are investigated. It has been found that ON-OFF control is only effective with the measurements of the relative speed of the DVA and the displacement of the basic design.

\section{References}

Allen, F. \& Karjalainen, R. (1999). Using genetic algorithms to find technical trading rules. Journal of Financial Economics, 51(2), 245-271.

Casciati, F., Magonette G. \& Marazzi F. (2006). Technology of semiactive devices and applications in vibration mitigation. New York: Wiley.

Diveyev, B., Horbay, O., Kernytskyy, I., Pelekh, R. \& Velhan, I. (2017). Dynamic properties and damping predictions for laminated micro-beams by different boundary conditions. In 13th International Conference on Perspective Technologies and Methods in MEMS Design: Lviv 20-23 April 2017 (pp. 30-34). New York: IEEE.

Fowlkes, W.Y. \& Creveling, C.M. (1995). Engineering methods for robust product design: using Taguchi methods in technology and 
product development. Reading, MA: Addison-Wesley Publishing Company.

Hu, H. \& Jin, D. (1997). A semi-active vibration control strategy based on piecewise linear vibration absorbers. Journal of Vibration Engineering, 10(2), 125-130.

Kernytskyy, I., Diveyev, B., Horbaj, O., Hlobchak, M., Kopytko, M. \& Zachek, O. (2017). Optimization of the impact multi-mass vibration absorbers. Scientific Review Engineering and Environmental Sciences, 26(3), 394-400.

Koo, J.H. \& Ahmadian, M. (2004). In search of suitable control methods for semi-active tuned vibration absorbers. Journal of Vibration and Control, 10(2), 163-174.

Moutinho, C. (2015). Testing a simple control law to reduce broadband frequency harmonic vibrations using semi-active tuned mass dampers. Smart Materials and Structures, 24(5), 055007. https://doi.org/10.1088/0964$1726 / 24 / 5 / 055007$

Nagarajaiah, S. (2009). Adaptive passive, semiactive, smart tuned mass dampers: identification and control using empirical mode decomposition, Hilbert transform, and short-term Fourier transform. Structural Control and Health Monitoring: The Official Journal of the International Association for Structural Control and Monitoring and of the European Association for the Control of Structures, 16(7-8), 800-841.

Pelekh, Y., Konyk, I., Hlobchak, M., Cherchyk, G., Opalko, V. \& Diveyev, B. (2017). Optimization of the particle vibration absorbers as SDOF system. In 13th International Conference on Perspective Technologies and Methods in MEMS Design: Lviv 20-23 April 2017 (pp. 30-34). New York: IEEE.

Pinkaew, S.T. \& Fujino, Y. (2001). Effectiveness of semi-active tuned mass dampers under harmonic excitation. Engineering Structures, 23(7), 850-856.

Qian, X. \& Hu, H. (2001). A semi-active vibration absorber with an adjustable clearance and its realization. Journal of Vibration Engineering, 14(4), 378-381.

Seiler, G., Fischer, O. \& Huber, P. (2002). Semi-active MR dampers in TMD's for vibration control of footbridges: II. Numerical analysis and practical realization. Paris: Footbridge Conference.

Setareh, M., Ritchey, J.K., Murray, T.M., Koo, J.H. \& Ahmadian, M. (2007). Semiactive tuned mass damper for floor vibration control. Journal of Structural Engineering, 133(2), 242-250.

Shen, Y. \& Ahmadian, M. (2013). Nonlinear dynamical analysis on four semi-active dynamic vibration absorbers with time delay. Shock and Vibration, 20(4), 649-663.

Spencer Jr, B.F. \& Nagarajaiah, S. (2003). State of the art of structural control. Journal of Structural Engineering, 129(7), 845-856.

Weber, F. \& Distl, H. (2013). Real-time controlled tuned mass dampers for Wolgograd Bridge. Beton-und Stahlbetonbau, 108(6), 362-372.

Zang, C., Friswell, M.I. \& Mottershead J.E. (2005). A review of robust optimal design and its application in dynamics. Computers and Structures, 83(4-5), 315-326.

\section{Summary}

Optimization of the semi-active vibration absorbers. In this paper, an efficient numerical approach is proposed to maximize the minimal damping of modes in a prescribed frequency range for general viscous tuned-mass systems. Methods of decomposition and numerical synthesis are considered on the basis of the adaptive schemes. The influence of dynamic vibration absorbers and basic design elastic and damping properties is under discussion. A technique is developed to give the optimal DVA's for the elimination of excessive vibration in sinusoidal and impact forced system. One task of this work is to analyze parameters identification of the dynamic vibration absorber and the basic structure. The questions of robustness at optimization of DVA are considered. Different types of control management for semi-active DVA's are applied. Examples of DVA's practical implementation are presented. 
Authors' address:

Ivan Kernytskyy - corresponding author

(https://orcid.org/0000-0001-6084-1774)

Warsaw University of Life Sciences - SGGW

Institute of Civil Engineering

Department of Mechanics and Building

Structures

Nowoursynowska 159, 02-776 Warsaw

Poland

e-mail: ivankernytskyy@ukr.net

Volodymyr Snitynskyy

(https://orcid.org/0000-0001-9633-1004)

Bohdan Diveyev

(https://orcid.org/0000-0001-5184-499X)

Orest Horbay

(https://orcid.org/0000-0002-0915-5637)

Ruslan Humenuyk

(https://orcid.org/0000-0001-7511-3673)

Yaroslav Sholudko

(https://orcid.org/0000-0001-5588-0066) 